\title{
Efeito do Formato de Onda e Gás de Proteção sobre a Taxa de Fusão e Geometria do Cordão na Soldagem MIG/ MAG-PV
}

\section{(Effect of Waveform and Shielding Gas on Melting Rate and Bead Geometry for MIG/MAG-PV)}

\author{
Alexandre Saldanha do Nascimento ${ }^{1}$, Luiz Carlos de Menezes Junior ${ }^{1}$, Louriel O. Vilarinho ${ }^{1}$ \\ ${ }^{1}$ Universidade Federal de Uberlandia, Laprosolda, Brasil, vilarinho@mecanica.ufu.br
}

\begin{abstract}
Resumo
Dada a importância do uso da polaridade variável na soldagem MIG/MAG (MIG/MAG-PV), com grande potencial para a aplicação de revestimento e para a soldagem de passe de raiz, é fundamental investigar os efeitos de parâmetros do processo na formação do cordão e na taxa de fusão do arame-eletrodo. Esta última em especial, dada a maior taxa de fusão obtida pelo período de polaridade negativa. Desta forma, procurou-se avaliar o efeito de dois formatos de onda investigados anteriormente que possuem pausa de corrente antes e após o pulso principal do periodo de polaridade positiva, haja vista que estas condições se mostraram as mais estáveis. Investigouse também o efeito do tipo de gás de proteção utilizado sobre as características geométricas do cordão de solda (representadas pela largura, penetração e reforço) e taxas de fusão e deposição e o rendimento. As soldagens foram executadas em simples deposição sobre chapas de aço SAE 1020 pelo processo MIG/MAG-PV com 30 e 50\% de porcentagem de tempo com eletrodo em polaridade negativa. Os gases de proteção utilizados foram $\mathrm{Ar}+2 \% \mathrm{O} 2, \mathrm{Ar}+5 \% \mathrm{O} 2$, Ar+8\%CO2 e Ar+15\%CO2. Procurou-se manter o mesmo comprimento de arco em todos os testes, por meio da variação da velocidade de alimentação de arame. Além disto, foi mantida constante a relação velocidade de alimentação de arame pela velocidade de soldagem, com o objetivo de tentar manter o volume de metal depositado constante para comparação. Pode-se concluir que o melhor entendimento do comportamento da geometria em relação aos parâmetros e consumíveis utilizados auxiliará para garantir o perfil do cordão desejado de acordo com a aplicação. Aliado a isto, a análise da taxa de fusão do processo possibilita selecionar com mais coerência os parâmetros e consumíveis utilizados que forneçam menores perdas de material e maior produtividade.
\end{abstract}

Palavras-chave: Polaridade Variável; Formato de Onda; MIG/MAG-PV.

Abstract: Due to the importance of variable polarity in MIG/MAG welding (MIG/MAG-PV) with great potential for overlaying and root pass applications, it is fundamental to assess the effect of process parameters on the bead formation and wire-burn rate, specially the latter, since higher melting rate is achieved by this process during negative polarity. Therefore, this work aims to assess the effect of two different waveforms previously investigated, in which current pauses before and after the main pulse during positive polarity (this condition leads to better stability). The shielding gas effect was also investigated for the bead geometry (width, penetration and reinforcement), melting rate, deposition rate and deposition efficiency. Bead-on-plate weldments were carried out over SAE 1020 carbon steel with MIG/MAG-PV welding at 30 and $50 \%$ of time in negative polarity. The employed shielding gases were $\mathrm{Ar}+2 \% \mathrm{O} 2, \mathrm{Ar}+5 \% \mathrm{O} 2$, $A r+8 \% \mathrm{CO} 2$ and $\mathrm{Ar}+15 \% \mathrm{CO} 2$. The arc length was kept approximately the same during the runs by varying the wire-feed speed. Also, the relationship between wire-feed speed and travel speed was kept constant in order to keep the same amount of deposited material. It is possible to conclude that the better understanding on the relationship among bead geometry, parameters and consumables leads to assure a desired bead profile, in accordance with a given application. Moreover, the melting rate analysis allows selecting more coherent parameters and consumables to assure lower material losses and high productivity.

Key-words: Variable Polarity; Waveform; $M I G / M A G-P V$

\section{Introdução}

As vantagens do uso da polaridade variável (PV) na soldagem MIG/MAG (MIG/MAG-PV) têm sido relatadas na literatura [1-4] como proporcionando ao processo de baixo

(Recebido em 29/02/2012; Texto final em 07/03/2012).

Artigo originalmente publicado no CONSOLDA 2011 aporte térmico e alta taxa de fusão, com grande potencial para a aplicação de revestimento e para a soldagem de passe de raiz. Dada esta grande importância, é fundamental investigar os efeitos de parâmetros do processo na formação do cordão e na taxa de fusão do arame-eletrodo. Esta última em especial, dada a maior taxa de fusão obtida pelo período de polaridade negativa.

Neste contexto, este trabalho foca em duas abordagens, lideradas pelo efeito do gás de proteção e parâmetros do processo de soldagem MIG/MAG-PV sobre a taxa de fusão e geometria do cordão. Os parâmetros a serem investigados são o formato 
de onda, a porcentagem de tempo em que o eletrodo opera em polaridade negativa (\%EN) e velocidade de alimentação (Va).

Com relação ao efeito do gás de proteção, sabe-se que a função primária dos gases nos processos de soldagem a arco é de fornecer um meio adequado para a operação estável e sustentar um arco em baixa voltagem e proporcionar proteção da contaminação atmosférica. Secundariamente, mas igualmente importante, inclui a função de controlar a geometria do cordão de solda e as propriedades mecânicas [5]. O gás de proteção tem uma influência significativa sobre o perfil do cordão de solda e das características de fusão. A área fundida total é aumentada (em uma corrente equivalente) pelo uso de gases que aumentam a energia do arco.

Com relação à taxa de fusão, sabe-se que sistemas automatizados de soldagem têm recebido muita atenção, pois são altamente adequados tanto para aumentar a taxa de produção e qualidade e diminuir custo e tempo de produção de um produto desejado. A fim de obtê-los, é indispensável um controle total sobre os parâmetros relevantes do processo para obter a geometria do cordão necessária, que também se baseia na soldabilidade [5].

Desta forma, neste trabalho, a avaliação das soldas em simples deposição variando os gases e tipo de onda em MIG/ MAG-PV, em relação a suas características econômicas foi definida pela taxa de deposição, taxa de fusão e o rendimento de deposição. A taxa de deposição é a massa do metal de adição depositado por unidade de tempo, numa dada intensidade de corrente, com fator de operação igual a $100 \%$. A taxa de fusão é determinada pela massa de metal fundida por unidade de tempo. Já o rendimento de deposição é a razão entre a massa de metal de solda depositado e a massa consumida do consumível, ou seja, razão entre a taxa de deposição e a taxa de fusão.

A taxa de fusão (TF) é determinada pela massa de metal de arame-eletrodo fundida por unidade de tempo, já a taxa de deposição (TD) é definida como a massa do metal de adição depositada por unidade de tempo, por sua vez o rendimento de deposição (Ren) é dado pela razão entre a taxa de fusão e a taxa de deposição. Estas características econômicas podem ser calculadas através das Equações 1, 2 e 3, respectivamente.

$$
T F=3,6 \frac{\rho \ell}{t_{S}}
$$

Onde $\ell$ - Comprimento do arame consumido (m); $\rho$ Densidade linear do arame $(\mathrm{g} / \mathrm{m})$ e $\mathrm{t}_{\mathrm{s}}$ - Tempo de soldagem (s).

$T D=3,6 \frac{\left(M_{f}-M_{i}\right)}{t_{S}}$

Onde, $\mathrm{M}_{\mathrm{f}}$ - Massa final da junta depois da soldagem (g) eM - Massa inicial da junta antes da soldagem (g).
$\operatorname{Re} n=\left(\frac{T D}{T F}\right) \times 100$

Onde, TD - taxa de deposição $(\mathrm{kg} / \mathrm{h})$ e TF - Taxa de fusão $(\mathrm{kg} / \mathrm{h})$.

\section{Metodologia}

A partir de trabalhos anteriores do grupo Laprosolda (Centro para Pesquisa e Desenvolvimento de Processos de Soldagem) [710], buscou-se avaliar o efeito dos formatos de onda denominados $\mathrm{B} \mathrm{e} \mathrm{C}$ (Tabelas 1 e 2, respectivamente), que apresentaram melhor desempenho operacional nos referidos trabalhos, e do tipo de gás de proteção utilizado sobre as características geométricas (representadas pela largura, penetração e reforço) e econômicas (caracterizada pelas taxas de fusão e deposição e o rendimento) do cordão de solda.

As soldagens foram executadas em simples deposição sobre chapas de aço SAE 1020 pelo processo MIG/MAG-PV com 30 e $50 \% \mathrm{EN}$. Os gases de proteção utilizados foram $\mathrm{Ar}+2 \% \mathrm{O}_{2}$, $\mathrm{Ar}+5 \% \mathrm{O}_{2}, \mathrm{Ar}+8 \% \mathrm{CO}_{2}$ e $\mathrm{Ar}+15 \% \mathrm{CO}_{2}$. O melhor entendimento do comportamento da geometria em relação aos parâmetros e consumíveis utilizados auxiliará para garantir o perfil do cordão desejado de acordo com a aplicação. Aliado a isto, a análise de características econômicas do processo possibilita selecionar com mais coerência os parâmetros e consumíveis utilizados que forneçam menores perdas de material e maior produtividade de acordo com o perfil desejado.

Procurou-se manter o mesmo comprimento de arco em todos os testes (em torno de $6 \mathrm{~mm}$ ), através da variação da velocidade de alimentação de arame. Além disto, foi mantido constante a relação velocidade de alimentação de arame pela velocidade de soldagem $(\mathrm{Va} / \mathrm{Vs}=9$, considerando ambas em $\mathrm{m} / \mathrm{min})$, com o objetivo de tentar manter o volume de metal depositado constante para comparação. Para tratamento dos resultados foi realizada uma análise de variânica (ANOVA) com significância de $95 \%$.

Na Tabela 3 são apresentados os valores de corrente média e eficaz utilizados, além da velocidade de alimentação de arame e velocidade de soldagem para cada par formato de onda e \%EN utilizados e ainda de acordo com o gás de proteção. 
Tabela 1. Parâmetros utilizados para a onda do tipo B.

\begin{tabular}{|c|c|c|c|c|c|c|c|c|}
\hline \multicolumn{2}{|r|}{ Onda B } & $I_{p}(A)$ & $\mathbf{t}_{\mathrm{p}}(\mathrm{ms})$ & $I_{n}(A)$ & $\mathrm{t}_{\mathrm{n}}(\mathrm{ms})$ & $I_{b}(A)$ & $\mathbf{t}_{\mathrm{b}}(\mathrm{ms})$ & $\%$ EN \\
\hline \multicolumn{2}{|r|}{$\mathrm{Tp}$} & 300 & 4 & 198 & 2,6 & 40 & 2 & 30 \\
\hline $\begin{array}{ll}\mathrm{Ib} & \mathrm{T} \\
1 & \mathrm{Ip}\end{array}$ & & 300 & 4 & 198 & 6,0 & 40 & 2 & 50 \\
\hline & & 300 & 4 & 198 & 14,0 & 40 & 2 & 70 \\
\hline
\end{tabular}

Tabela 2. Parâmetros calculados para a onda do tipo C.

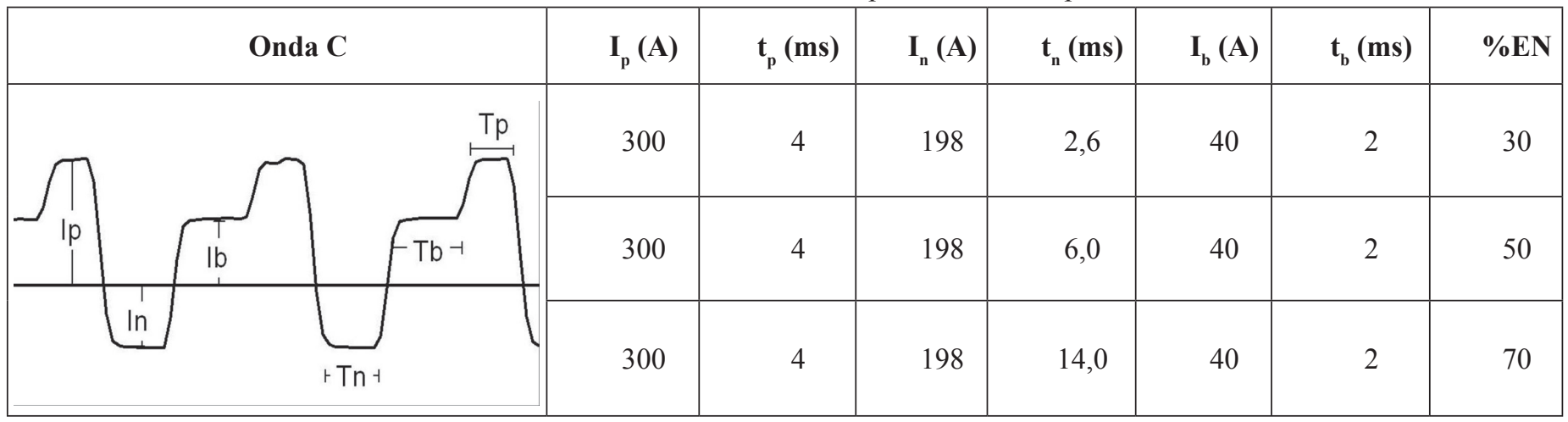

Tabela 3. Parâmetros utilizados em função do gás e tipo de onda.

\begin{tabular}{|c|c|c|c|c|c|}
\hline Gás & Onda \%EN & $\operatorname{Va}(\mathrm{m} / \mathrm{min})$ & Vs $(\mathrm{cm} / \mathrm{min})$ & $\mathrm{I}_{\mathrm{RMS}}(\mathrm{A})$ & $I_{\text {med }}(A)$ \\
\hline $\mathrm{Ar}+5 \mathrm{O}_{2}$ & В 30 & 5,8 & 38,6 & 232 & 208 \\
\hline $\mathrm{Ar}+5 \mathrm{O}_{2}$ & В 50 & 6,3 & 42,0 & 223 & 205 \\
\hline $\mathrm{Ar}+5 \mathrm{O}_{2}$ & C 30 & 5,9 & 39,3 & 232 & 208 \\
\hline $\mathrm{Ar}+5 \mathrm{O}_{2}$ & C 50 & 6,3 & 42,0 & 223 & 205 \\
\hline $\mathrm{Ar}+2 \mathrm{O}_{2}$ & B 30 & 6,0 & 39,6 & 232 & 208 \\
\hline $\mathrm{Ar}+2 \mathrm{O}_{2}$ & В 50 & 6,6 & 44,0 & 223 & 205 \\
\hline $\mathrm{Ar}+2 \mathrm{O}_{2}$ & C 30 & 6,2 & 41,3 & 232 & 208 \\
\hline $\mathrm{Ar}+2 \mathrm{O}_{2}$ & C 50 & 6,6 & 44,0 & 223 & 205 \\
\hline $\mathrm{Ar}+8 \mathrm{CO}_{2}$ & B 30 & 5,0 & 33,0 & 215 & 183 \\
\hline $\mathrm{Ar}+8 \mathrm{CO}_{2}$ & В 50 & 4,8 & 32,0 & 191 & 163 \\
\hline $\mathrm{Ar}+8 \mathrm{CO}_{2}$ & C 30 & 5,2 & 34,6 & 215 & 183 \\
\hline $\mathrm{Ar}+8 \mathrm{CO}_{2}$ & C 50 & 5,0 & 33,3 & 191 & 163 \\
\hline $\mathrm{Ar}+15 \mathrm{CO}_{2}$ & B 30 & 4,8 & 32,0 & 221 & 193 \\
\hline $\mathrm{Ar}+15 \mathrm{CO}_{2}$ & B 50 & 4,9 & 32,6 & 203 & 180 \\
\hline $\mathrm{Ar}+15 \mathrm{CO}_{2}$ & C 30 & 4,9 & 32,6 & 221 & 193 \\
\hline $\mathrm{Ar}+15 \mathrm{CO}_{2}$ & C 50 & 5,0 & 33,3 & 203 & 180 \\
\hline
\end{tabular}




\section{Resultados e Discussão}

\subsection{Resultados para características geométricas}

Na Tabela 4 são dispostos os resultados dos valores médios medidos das características geométricas (largura, penetração e reforço) dos cordões de solda obtidos. Já a Figura 1 apresenta o comportamento do reforço do cordão de solda em função do tipo de onda e \%EN. A partir desta figura é possível perceber que existe a tendência de reforços do cordão maiores a medida que se aumenta o \% EN para os dois formatos de onda estudados, resultando em cordões mais convexos. Este resultado está de acordo com trabalhos anteriores e já era esperado, visto que com o aumento do \%EN, é aumentado também o tempo em que o processo opera na polaridade negativa, com isto o calor do arco fica mais tempo concentrado no eletrodo, resultando em menor molhabilidade do metal base ao mesmo tempo em que se aumenta o volume de metal depositado devido a maior taxa de fusão e consequentemente maiores reforços são gerados. De toda forma, apesar de elevados valores de reforço não serem atraentes em diversas aplicações de soldagem, no caso de juntas sobrepostas, por exemplo, esta característica é favorável a maior adaptabilidade a aberturas na junta.

Tabela 4. Resultados de características geométricas.

\begin{tabular}{|c|r|r|r|r|}
\hline Gás & $\begin{array}{c}\text { Onda } \\
\mathbf{\%} \mathbf{E N}\end{array}$ & $\begin{array}{r}\mathbf{L} \\
(\mathbf{m m})\end{array}$ & $\begin{array}{r}\mathbf{P} \\
(\mathbf{m m})\end{array}$ & $\begin{array}{r}\mathbf{R} \\
(\mathbf{m m})\end{array}$ \\
\hline $\mathrm{Ar}+5 \mathrm{O}_{2}$ & B 30 & 8,82 & 2,34 & 2,65 \\
\hline $\mathrm{Ar}+5 \mathrm{O}_{2}$ & B 50 & 6,92 & 1,23 & 2,32 \\
\hline $\mathrm{Ar}+5 \mathrm{O}_{2}$ & C 30 & 8,49 & 2,32 & 2,13 \\
\hline $\mathrm{Ar}+5 \mathrm{O}_{2}$ & C 50 & 8,12 & 1,30 & 2,46 \\
\hline $\mathrm{Ar}+2 \mathrm{O}_{2}$ & B 30 & 8,87 & 1,39 & 2,61 \\
\hline $\mathrm{Ar}+2 \mathrm{O}_{2}$ & B 50 & 7,58 & 0,98 & 2,49 \\
\hline $\mathrm{Ar}+2 \mathrm{O}_{2}$ & C 30 & 8,10 & 1,89 & 2,40 \\
\hline $\mathrm{Ar}+2 \mathrm{O}_{2}$ & C 50 & 7,48 & 1,33 & 2,63 \\
\hline $\mathrm{Ar}+8 \mathrm{CO}_{2}$ & B 30 & 8,15 & 2,59 & 2,34 \\
\hline $\mathrm{Ar}+8 \mathrm{CO}_{2}$ & B 50 & 7,71 & 1,80 & 2,53 \\
\hline $\mathrm{Ar}+8 \mathrm{CO}_{2}$ & C 30 & 8,04 & 1,94 & 2,32 \\
\hline $\mathrm{Ar}+8 \mathrm{CO}_{2}$ & C 50 & 8,17 & 2,53 & 2,44 \\
\hline $\mathrm{Ar}+15 \mathrm{CO}_{2}$ & B 30 & 7,25 & 1,36 & 2,49 \\
\hline $\mathrm{Ar}+15 \mathrm{CO}_{2}$ & B 50 & 7,95 & 1,82 & 2,48 \\
\hline $\mathrm{Ar}+15 \mathrm{CO}_{2}$ & C 30 & 9,08 & 1,89 & 2,30 \\
\hline $\mathrm{Ar}+15 \mathrm{CO}_{2}$ & C 50 & 8,70 & 1,97 & 2,16 \\
\hline
\end{tabular}

O efeito do tipo de gás de proteção utilizado sobre o reforço do cordão de solda é apresentado na Figura 2. É verificado que ao se aumentar o teor de $\mathrm{O}_{2}$ de $2 \%$ para $5 \%$ o reforço do cordão é reduzido. O mesmo comportamento é percebido com o uso de $\mathrm{CO}_{2}$, onde os menores reforços aconteceram para o teor mais elevado deste gás.

Segundo Pires et al. [11], a temperatura do arco deve ser maior para misturas com mais alto teor de $\mathrm{O}_{2}$, especialmente na vizinhança do eletrodo devido a reações exotérmicas entre $\mathrm{O}_{2}$ e elementos do arame eletrodo e a poça de fusão, especialmente ferro e carbono. Além deste efeito de aumento de temperatura, há outro ponto talvez até mais importante a ser condiserado, que se refere à condutividade térmica. Tanto o $\mathrm{O}_{2}$ quanto o $\mathrm{CO}_{2}$ possuem maior condutividade térmica que o Ar (condutividade térmica $\mathrm{Ar}=0,02573 \mathrm{~W} /(\mathrm{m} . \mathrm{K}) ; \mathrm{O}_{2}=0,04191 \mathrm{~W} /(\mathrm{m} . \mathrm{K})$ e $\mathrm{CO}_{2}$ $=0,03293 \mathrm{~W} /(\mathrm{m} . \mathrm{K})$, todos a 1 atm e $500 \mathrm{~K}[12])$. Esta maior condutividade térmica implica numa maior entrega térmica à poça de fusão. Além disto, maiores teores de $\mathrm{O}_{2}$ implicam em maior fluidez do metal de solda e maior molhabilidade do metal de base, ocasionando o menor reforço.

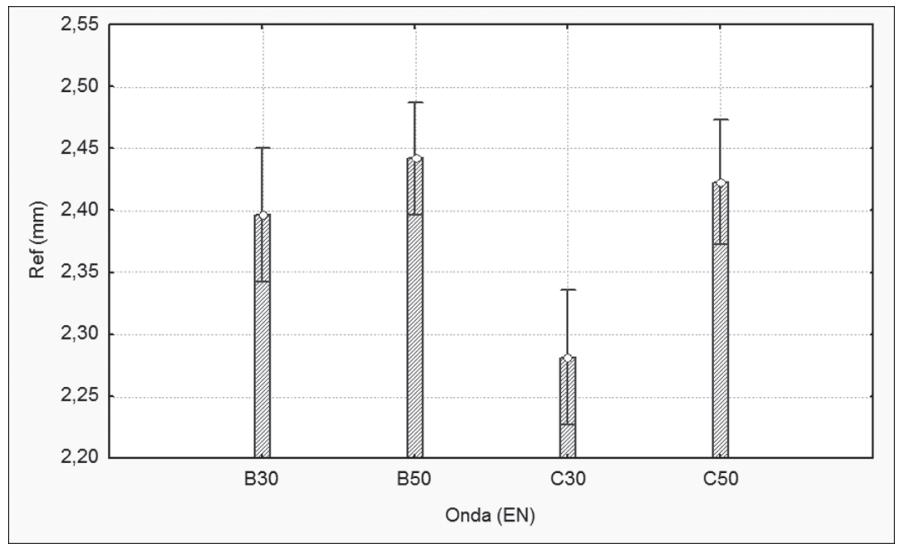

Figura 1. Reforço em função do tipo de onda e do \%EN.

Relativamente a Figura 2, a porcentagem de $\mathrm{CO}_{2}$ na mistura binária Argônio/ $\mathrm{CO}_{2}$, pode-se observar a diminuição do reforço a medida que a porcentagem de $\mathrm{CO}_{2}$ no gás de proteção se eleva. Este efeito se deve aos fatores discutidos no parágrafo anterior, que promovem o "espalhamento" do metal de solda sobre o metal base e a consequente redução do reforço.

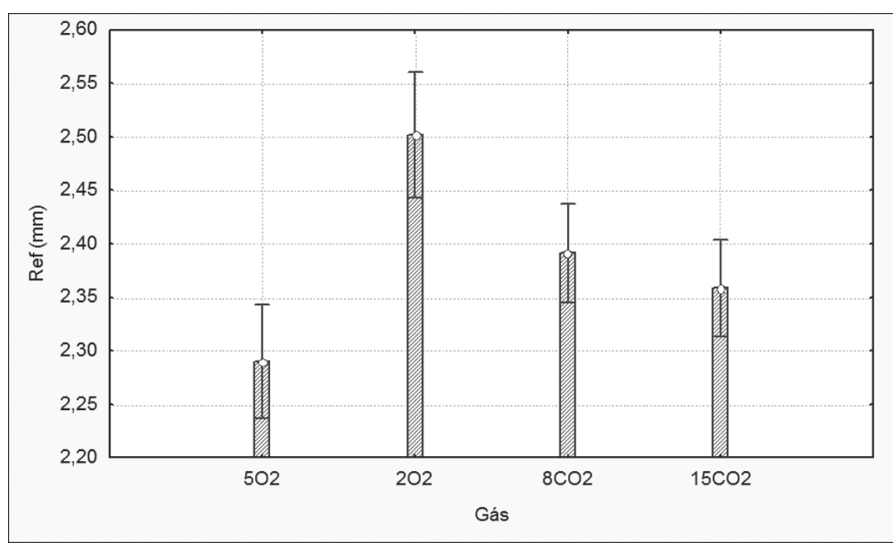

Figura2 - Reforço em função do tipo de gás de proteção.

Em relação a Figura 3 referente ao comportamento da penetração em função do tipo de onda e do \% EN, verifica-se que ocorre a tendência de a penetração diminuir com o aumento de $\%$ EN para os dois formatos de onda estudados. Este resultado está de acordo com o encontrado por diversos autores [13], pois o maior tempo de permanência do eletrodo em polaridade negativa faz com que ocorra a redução do aporte térmico sobre 
o metal base e consequentemente redução da penetração. Além disto, pode-se perceber que os resultados para o tipo de onda $\mathrm{C}$ que possui período de base positiva antes do pulso de destacamento apresentou maiores valores de penetração quando comparado ao formato de onda B com base positiva após o pulso de destacamento. A razão para isto pode ser atribuída ao fato de que no formato de onda $\mathrm{C}$, a gota metálica é destacada ao final do pulso positivo e é transferida no momento em que ocorre a inversão de polaridade, ou seja, atravessa o arco sobre ação das forças repulsivas típicas do uso de eletrodo negativo, permanecendo mais tempo no arco sobre correntes mais elevada e aquecendo mais. Em razão disto, resulta em maior penetração do que os cordões de solda resultantes da onda B, onde as gotas atravessam mais rapidamente e sobre efeito da corrente de base positiva que é inferior a corrente negativa utilizada.

De acordo com Jelmorini et al. [14] a temperatura das gotas durante a transferência metálica no processo MIG/MAG pode variar entre $1800 \mathrm{e} 2400^{\circ} \mathrm{C}$, aumentando diretamente com o valor da corrente em uma faixa de 100 a 400 A. Em uma avaliação mais recente, Yamazaki et al. [15] estimam a temperatura da gota entre 2000 e $2160{ }^{\circ} \mathrm{C}$ para uma faixa de corrente de 225 a 350 A, também em MIG/MAG.

Desta forma, apesar de a corrente média ser a mesma nos formatos de onda $\mathrm{B}$ e $\mathrm{C}$, no momento da transferência a gota metálica experimenta maior valor de corrente na forma de onda $\mathrm{C}$, justificando seu maior aquecimento neste momento e implicando em maior penetração.

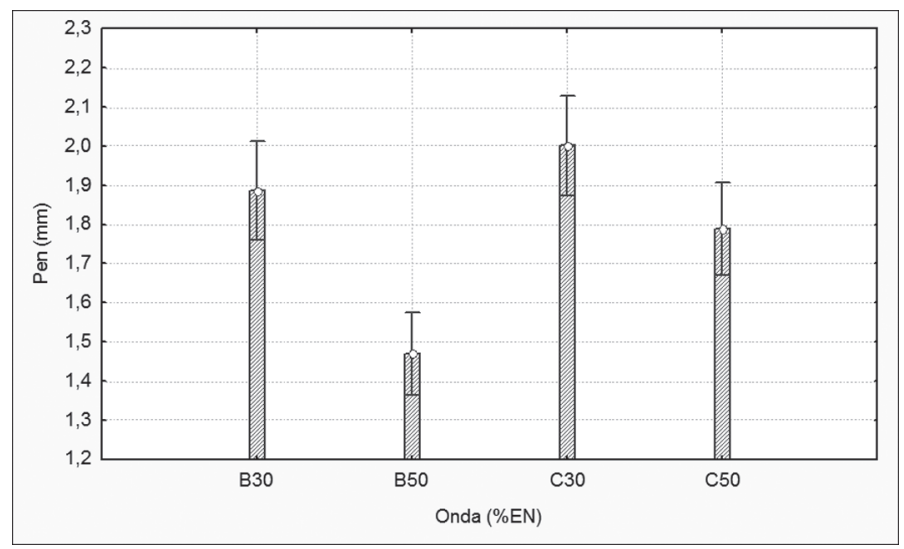

Figura 3. Penetração em função do tipo de onda e do \%EN.

Na Figura 4 é mostrado o comportamento da penetração em função do tipo de gás de proteção empregado. O efeito do aumento do potencial de oxigênio do gás de proteção, decorrente da introdução de uma maior porcentagem de $\mathrm{CO}_{2}$ ou $\mathrm{O}_{2}$ na mistura binária baseada em Argônio, também pode ser observado ao se analisar a Figura 4. A partir desta figura, pode-se verificar um aumento na penetração do cordão de solda em razão, provavelmente, de uma maior temperatura do arco, deste modo, facilitando a diluição do metal de adição no metal de solda. Com exceção da condição onde se utilizou o gás Ar$15 \% \mathrm{CO}_{2}$ que forneceu menor penetração quando comparado ao gás Ar- $8 \% \mathrm{CO}_{2}$, este resultado pode ser explicado pela maior repulsão da gota (diminuindo a parcela do efeito mecânico da quantidade de movimento da transferência na penetração), maior instabilidade do processo, implicando em muitos respingos e eventuais curto-circuitos quando do uso de $\mathrm{Ar}-15 \mathrm{CO}_{2}$, o que, ao diminuir o comprimento do arco, pode-se reduzir o aporte térmico (o curto-circuito reduz a energia de soldagem ao reduzir diretamente o valor da tensão, mas não necessariamente o quanto de calor se entrega para a peça).

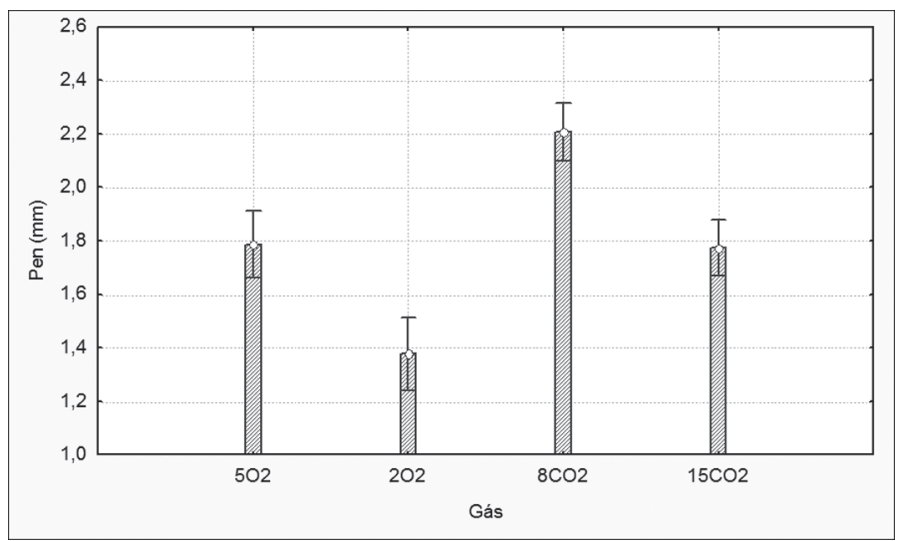

Figura 4. Penetração em função do tipo de gás de proteção.

Para os dois formatos de onda propostos, a largura do cordão de solda diminuiu com o aumento do \%EN, como mostrado na Figura 5, apresentando tendência a cordões mais convexos com aumento de reforço e redução da largura, comportamento típico do processo MIG/MAG-PV, pois como já visto anteriormente, nestas condições o aporte de calor é em sua maior parte direcionado para o arame-eletrodo aquecendo menos a chapa e com isto ocorre a redução do molhamento do metal de base. Entretanto, mesmo utilizando valores iguais de Corrente Eficaz nos dois formatos de onda e mesmo comprimento de arco, o formato de onda $\mathrm{C}$, como abordado anteriormente a respeito da transferência metálica e maior aquecimento da gota, fornece maiores valores de largura do cordão de solda e menores reforços, reduzindo a convexidade dos cordões, sendo mais vantajoso na maioria das aplicações.

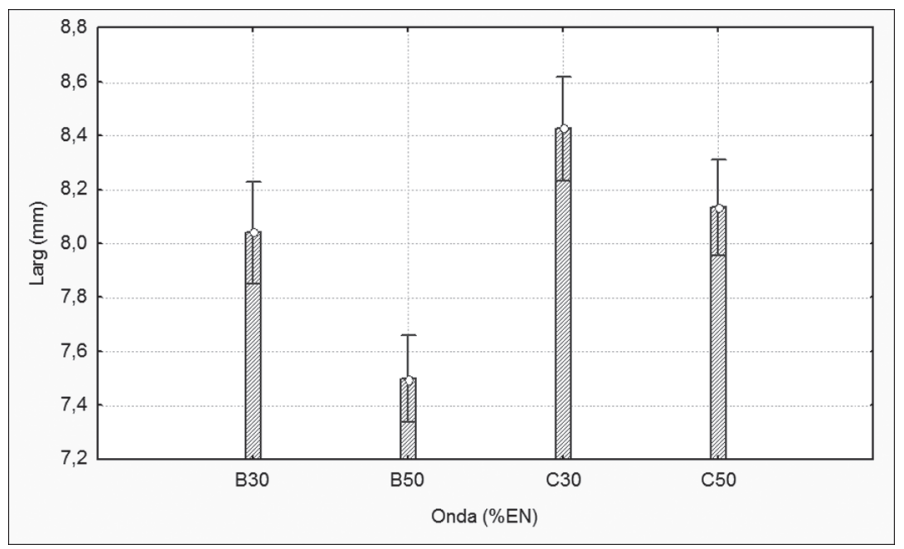

Figura 5. Largura em função do tipo de onda e do \%EN.

Comparando os gases com adição de oxigênio, não houve diferença significativa na largura, como pode ser visualizado na Figura 6. No entanto, o maior aquecimento pelos fatores discutidos (condutividade térmica e reação exotérmica de 
oxidação) com adição de $15 \% \mathrm{CO}_{2}$ forneceu a maior largura de cordão (maior molhamento).

Na maioria das aplicações em soldagem, é preferível cordões de solda mais planos e menos convexos, pois conseqüentemente melhoram em resistência a fadiga, assim como em redução de custos. No entanto, em certos casos específicos, cordões com maiores reforços são de interesse por aumentarem a adaptabilidade a aberturas de juntas.

A partir dos resutados obtidos, é possível concluir que o perfil do reforço e largura, em geral podem ser melhorados (reduzindo a convexidade), com o aumento do potencial de oxigênio da mistura gasosa e/ou com a utilização do formato de onda do tipo $\mathrm{C}$ que possui base positiva antes do pulso de destacamento, onde nestas condições se alcança maior molhabilidade do metal de base. Em todo caso, como citado anteriormente, em algumas aplicações, como em juntas sobrepostas com abertura entre as chapas, ou situações em que ocorre deformação da junta devido o aporte de calor e consequente aumento da abertura de raiz das juntas, cordões com elevados reforços podem ser mais eficazes na adaptabilidade a tais tipos de união.

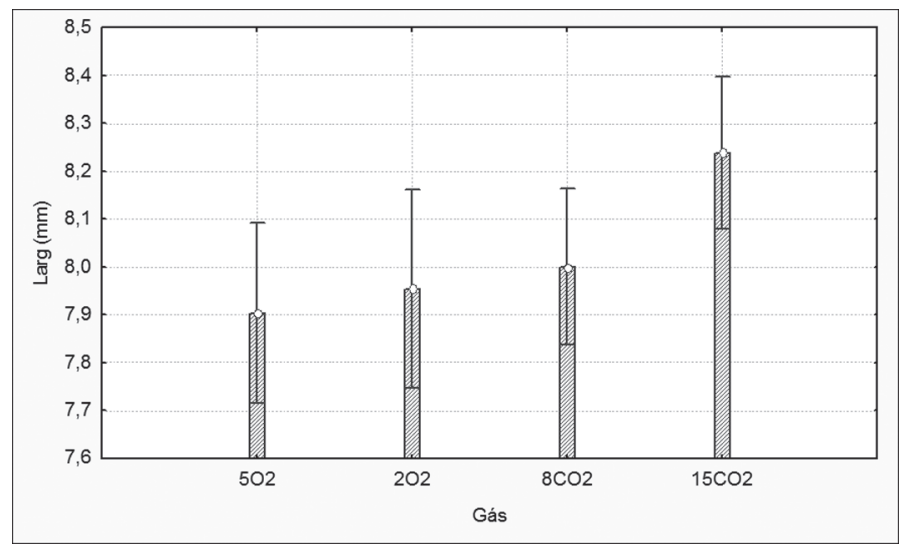

Figura 6. Largura em função do tipo de gás de proteção.

Na Tabela 5 são apresentados os valores obtidos através de análise de variância (ANOVA) dos resultados para uma confiabilidade de $95 \%$. Foi verificado que os fatores gás de proteção e tipo de onda de corrente utilizados, assim como a interação destes fatores, exercem influência estatística significativa sobre os resultados de características geométricas nas condições estudadas.

Tabela 5. Valores obtidos através da análise estatística de variância dos resultados.

\begin{tabular}{|c|c|c|c|}
\hline Parâmetro & Reforço & Penetração & Largura \\
\hline Gás & 0,000028 & 0,000000 & 0,035125 \\
\hline Onda & 0,000245 & 0,000000 & 0,000000 \\
\hline Interação & 0,000001 & 0,000000 & 0,000000 \\
\hline
\end{tabular}

\subsection{Resultados para características econômicas}

Os resultados médios dos valores calculados das características econômicas estão dispostos na Tabela 6 para as condições estudadas, calculados conforme as Equações 1 a 3.
Tabela 6. Resultados médios de características econômicas.

\begin{tabular}{|c|c|c|c|c|}
\hline Gás & $\begin{array}{l}\text { Onda } \\
\% \text { EN }\end{array}$ & TF (kg/h) & TD $(\mathrm{kg} / \mathrm{h})$ & $\operatorname{Ren}(\%)$ \\
\hline $\mathrm{Ar}+5 \mathrm{O}_{2}$ & B 30 & 3,22 & 3,15 & 97,88 \\
\hline $\mathrm{Ar}+5 \mathrm{O}_{2}$ & B 50 & 3,56 & 2,80 & 78,65 \\
\hline $\mathrm{Ar}+5 \mathrm{O}_{2}$ & C 30 & 3,32 & 3,15 & 94,90 \\
\hline $\mathrm{Ar}+5 \mathrm{O}_{2}$ & C 50 & 3,58 & 3,31 & 92,40 \\
\hline $\mathrm{Ar}+2 \mathrm{O}_{2}$ & В 30 & 3,12 & 2,84 & 91,10 \\
\hline $\mathrm{Ar}+2 \mathrm{O}_{2}$ & B 50 & 3,40 & 2,63 & 77,46 \\
\hline $\mathrm{Ar}+2 \mathrm{O}_{2}$ & C 30 & 3,19 & 3,03 & 94,90 \\
\hline $\mathrm{Ar}+2 \mathrm{O}_{2}$ & C 50 & 3,40 & 3,04 & 89,50 \\
\hline $\mathrm{Ar}+8 \mathrm{CO}_{2}$ & B 30 & 2,70 & 2,52 & 93,42 \\
\hline $\mathrm{Ar}+8 \mathrm{CO}_{2}$ & B 50 & 2,60 & 2,43 & 93,54 \\
\hline $\mathrm{Ar}+8 \mathrm{CO}_{2}$ & C 30 & 2,80 & 2,62 & 93,36 \\
\hline $\mathrm{Ar}+8 \mathrm{CO}_{2}$ & C 50 & 2,70 & 2,51 & 93,00 \\
\hline $\mathrm{Ar}+15 \mathrm{CO}_{2}$ & В 30 & 2,60 & 2,23 & 86,00 \\
\hline $\mathrm{Ar}+15 \mathrm{CO}_{2}$ & B 50 & 2,62 & 2,21 & 84,30 \\
\hline $\mathrm{Ar}+15 \mathrm{CO}_{2}$ & C 30 & 2,62 & 2,43 & 92,77 \\
\hline $\mathrm{Ar}+15 \mathrm{CO}_{2}$ & C 50 & 2,70 & 2,53 & 93,57 \\
\hline
\end{tabular}

Na Figura 7 é apresentado o resultado da taxa de fusão para os gases estudados. Pode-se observar que os maiores valores foram obtidos para os gases com adição de oxigênio, ao contrário do que era de se esperar, pois o $\mathrm{CO}_{2}$ quando adicionado ao gás de proteção possui a característica de fornecer arco mais quente (reações exotérmicas) e com maior condutividade térmica. Entretanto, nas condições utilizadas neste trabalho, em que a metodologia de análise em volume constante foi adotada, esta premissa foi prejudicada, já que a corrente média de soldagem utilizada nos gases com adição de $\mathrm{O}_{2}$ foram maiores do que quando do uso de $\mathrm{CO}_{2}$ (variando de 15 a $45 \mathrm{~A}$ mais altas, conforme apresentado na Tabela 6), suprimindo o efeito de maior aquecimento do $\mathrm{CO}_{2}$. Ressalta-se que justamente por proporcionar arcos mais quentes, pela metodologia para determinação de parâmetros adotada neste trabalho, quando se utiliza adição de $\mathrm{CO}_{2}$ na mistura gasosa, menores valores de corrente negativa são necessários para igualar a taxa de fusão em polaridade positiva. Por isso a diferença nos valores de corrente média utilizados e consequentemente a utilização de velocidades de alimentação distintas para se manter o mesmo comprimento de arco. No entanto, estes valores foram corrigidos através da velocidade de soldagem para manter o mesmo volume de material por unidade de comprimento. 


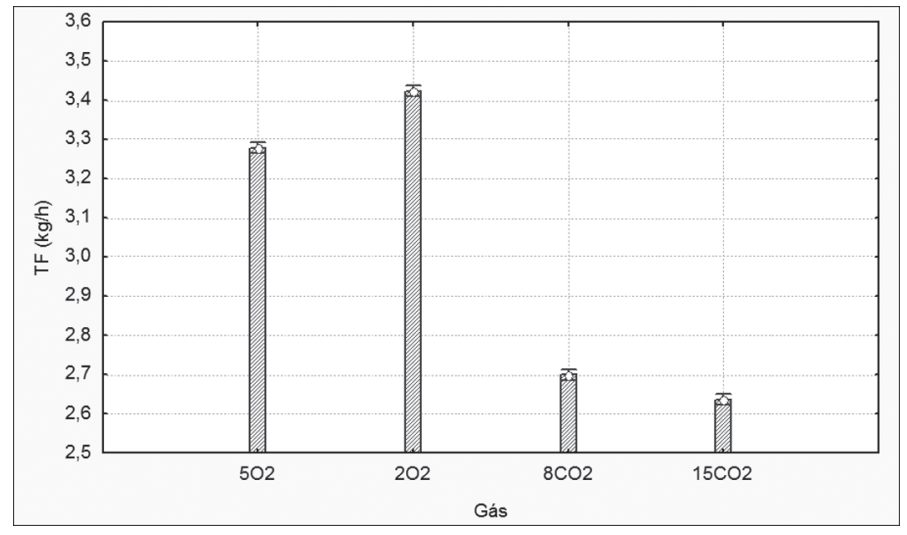

Figura 7. Taxa de fusão em função do gás de proteção.

Em relação ao tipo de onda e \%EN, de acordo com a Figura 8 a taxa de fusão do arame-eletrodo se torna maior com o aumento do $\% \mathrm{EN}$, resultado que está de acordo com o divulgado por diversos autores e é comportamento típico do processo MIG/MAG-PV, pois nestas condições, o maior tempo em que o eletrodo opera em polaridade negativa quando se aumenta o \%EN é responsável pelo seu maior aquecimento, resultando em maior taxa de fusão. Além disto, ao se comparar o formato de onda, é possível perceber que o tipo de onda $\mathrm{C}$ forneceu maiores valores de taxa de fusão. Sugere-se que tal característica é devido ao fato de que a pausa após o pulso reduz o aquecimento do arame quando ele está quente. Se a pausa for no início o aquecimento é mais lento inicialmente, mas se mantém até o final. Lembrando que termicamente pela difusão de calor, o aquecimento é, por natureza, mais lento que o resfriamento. Assim, a pausa no início contribui menos para o retardo do aquecimento, do que uma pausa no final que contribuiria mais para o resfriamento. A Figura 9 procura ilustrar esta explicação.

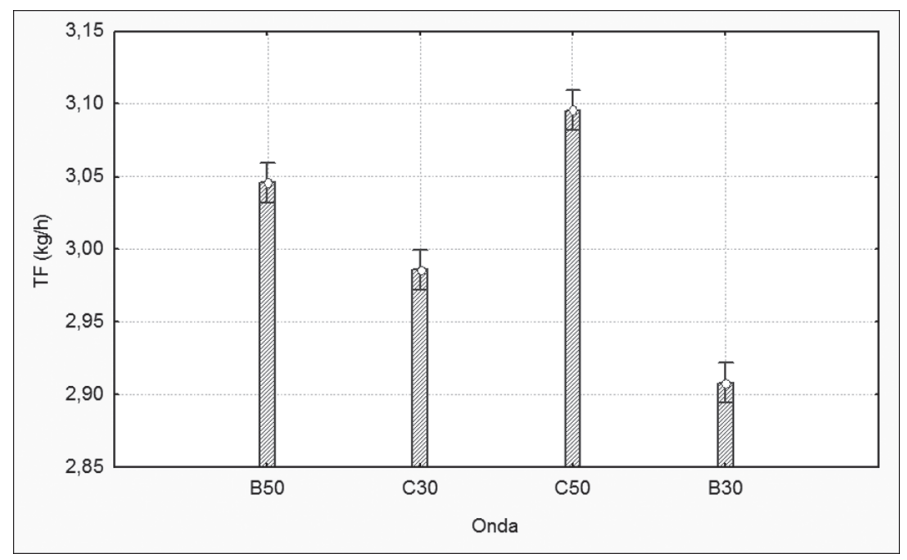

Figura 8. Taxa de fusão em função do formato de onda e $\%$ EN.

Outro critério muito importante na seleção de determinado processo de soldagem, consumíveis e parâmetros adotados, seria a taxa de deposição, pois nem todo material que é fundido é adicionado a junta, ocorrendo perdas por respingos (ou salpicos), além da evaporação metálica. Neste sentido, nas Figuras 10 e 11, são apresentados os efeitos do tipo de gás de proteção e do formato de onda e \%EN no processo MIG/MAGPV, respectivamente, sobre a taxa de deposição.

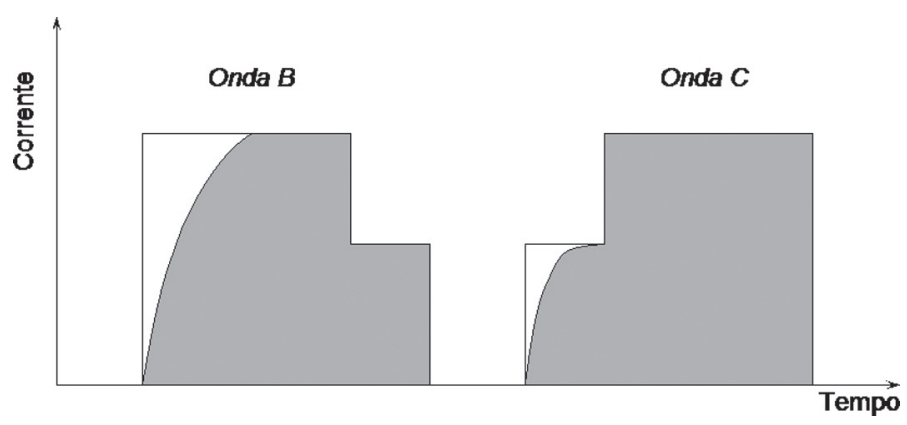

Figura 9. Proposta esquemática para maior taxa de fusão da onda $\mathrm{C}$ em comparação à onda $\mathrm{B}$. A parte sombreada representaria uma integral da energia, equivalente ao aquecimento do arame.

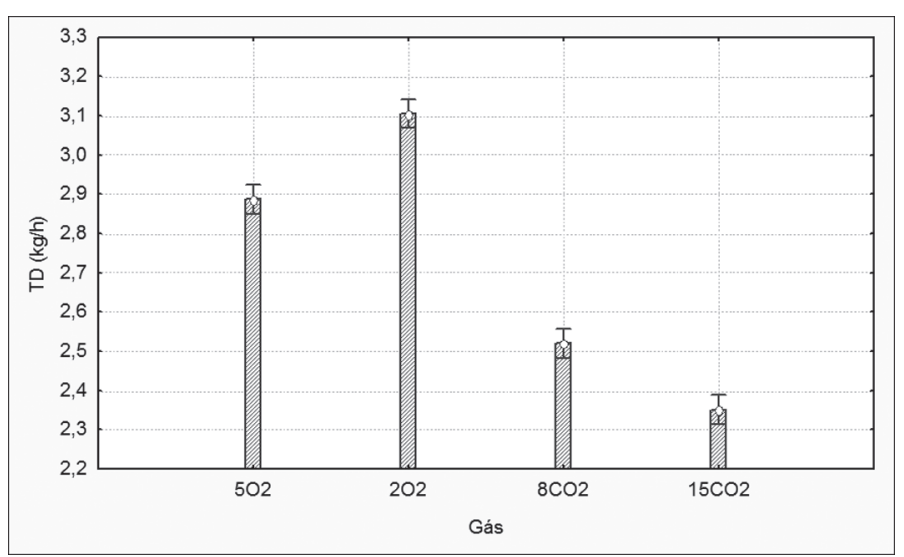

Figura 10. Taxa de deposição em função do tipo de gás de proteção.

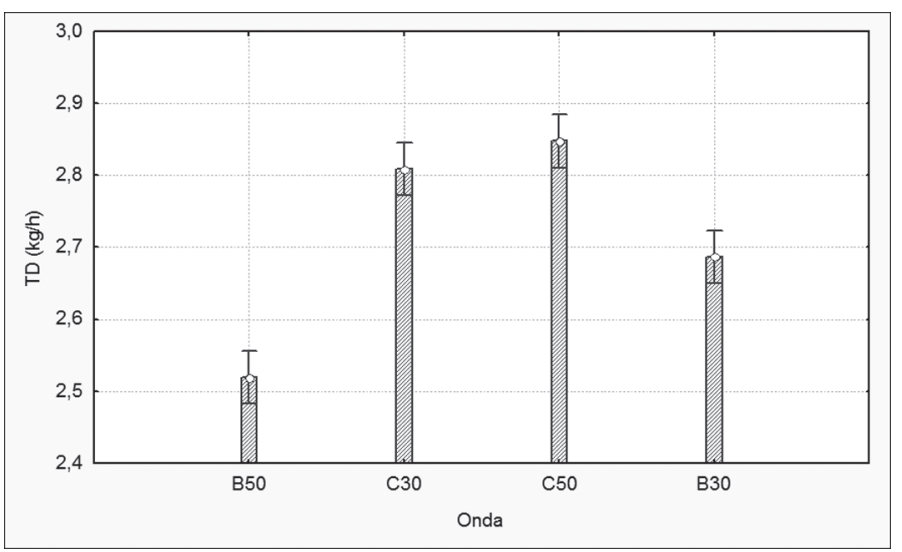

Figura 11. Taxa de deposição em função do formato de onda e \%EN.

Na Figura 10, é verificado que a maior taxa de deposição foi alcançada quando o gás $\mathrm{Ar}-2 \% \mathrm{O}_{2}$ foi utilizado, nesta atmosfera protetora, os melhores resultados de estabilidade de arco e de transferência metálica foram obtidos, desta forma, resultando em menores perdas e maior deposição. Já para os gases com 
adição de $\mathrm{CO}_{2}$ na mistura foram observados os menores valores de taxa de deposição, pois como discutido anteriormente, na metodologia utilizada, menores valores de corrente média foram utilizados quando comparados com os executados em atmosfera de $\mathrm{Ar}-\mathrm{O}_{2}$. Portanto, como as taxas de fusão foram menores para $\mathrm{Ar}-\mathrm{CO}_{2}$, devido às menores correntes, suprimindo o efeito de maiores temperaturas em atmosfera com $\mathrm{CO}_{2}$ devido as reações exotérmicas, a taxa de deposição acompanhou este comportamento.

Na Figura 11 são apresentados os resultados de taxa de deposição em função dos formatos de onda e \% $\mathrm{EN}$ avaliados. Como afirmado anteriormente na metodologia para determinação de parâmetros, o efeito da "suavização" da transferência, devido a desaceleração da gota, no momento em que atravessa o arco, que ocorre no formato de onda $\mathrm{C}$ refletiu em arcos mais estáveis e com transferência mais regular, consequentemente menores perdas foram observadas, justificando, portanto, as mais altas taxas de deposição quando da utilização deste formato de onda. Foi observado ainda um pequeno acréscimo na taxa de deposição com o aumento do \%EN para o tipo de onda $\mathrm{C}$, acompanhando o comportamento da taxa de fusão para esta mesma condição.

Já nos formatos de onda B, ainda avaliando a Figura 11, houve redução da taxa de deposição com o aumento do \%EN ao contrário do que foi observado para a taxa de fusão, este resultado sugere que, de modo geral, ao se elevar o \% $\mathrm{EN}$ maiores perdas são experimentadas para este formato de onda, sendo mais susceptíveis aos efeitos de repulsão da gota e instabilidades características do uso de polaridade negativa.

Finalmente, nas Figuras 12 e 13 são apresentados os efeitos do tipo de gás de proteção e do formato de onda e \%EN em MIG/MAG-PV, sobre o rendimento de deposição do processo. Este parâmetro indica entre as condições utilizadas qual fornece o melhor conjunto de parâmetros que forneça as menores perdas de material.

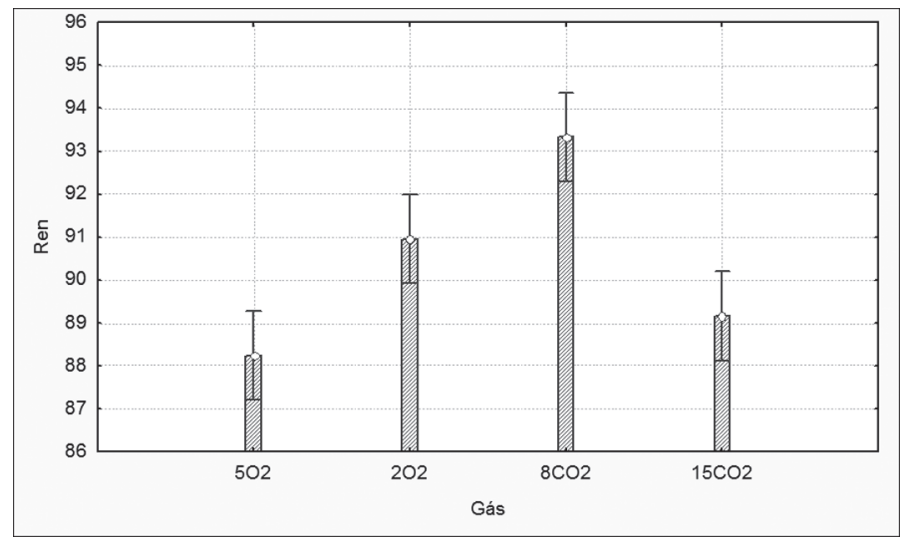

Figura 12. Rendimento de deposição em função do tipo de gás de proteção.

De acordo com os resultados obtidos, analisando a Figura 12 , os maiores rendimentos do processo foram obtidos nas condições onde se utilizou o gás com $8 \% \mathrm{CO}_{2}$ adicionado. Ao contrário do que se esperava, já que a maior estabilidade de arco foi obtida com Ar- $2 \% \mathrm{O}_{2}$, este resultado pode ser justificado, talvez pelos maiores níveis de corrente média ao se utilizar $\mathrm{O}_{2}$ podendo ter intensificado as perdas por evaporação metálica. De qualquer forma, os valores de rendimento encontrados foram satisfatórios com o menor deles próximo de $90 \%$.

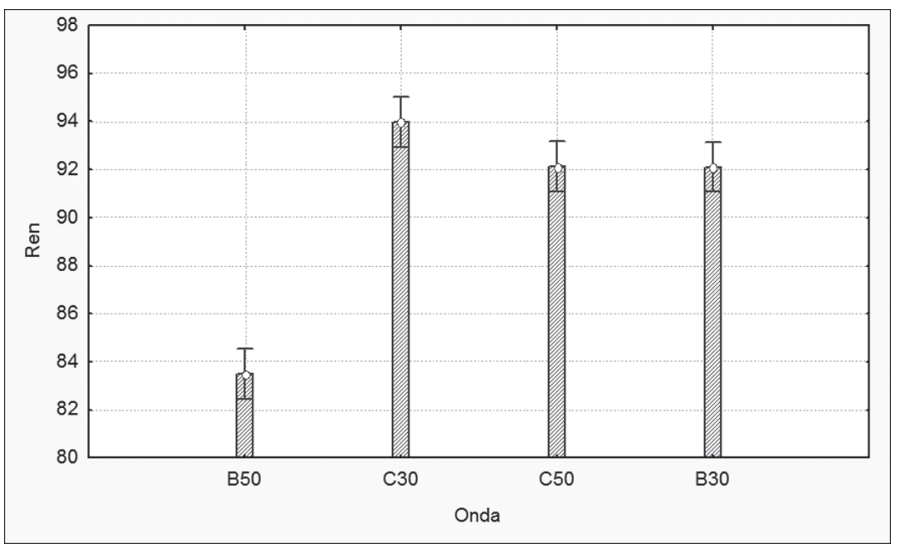

Figura 13. Rendimento de deposição em função do formato de onda e \%EN.

É possível perceber que, de acordo com a Figura 13, há a tendência de reduzir o rendimento de deposição a medida que se aumenta o \%EN em MIG/MAG-PV. Possivelmente, este comportamento ocorre devido o aumento do período em que o eletrodo opera em polaridade negativa gerando mais perdas devido a maior instabilidade nestas condições. Foi verificado ainda que, de modo geral, os maiores rendimentos de deposição foram encontrados para as condições onde se utilizou o formato de onda $\mathrm{C}$, confirmando sua maior estabilidade e regularidade na transferência metálica.

A Tabela 7 apresenta os resultados de análise de variância, ANOVA, para uma confiabilidade de $95 \%$, sobre os resultados de características econômicas. Nas condições adotadas neste trabalho, os parâmetros gás de proteção, formato de onda e \%EN, assim como a interação entre eles exercem influência estatística significativa sobre os resultados de taxa de fusão (TF), taxa de deposição (TD) e rendimento (Ren) estudados.

Tabela 7. Valores obtidos através da analise estatística de variância dos resultados de características econômicas.

\begin{tabular}{|c|c|c|c|}
\hline Parâmetro & TF & TD & Ren \\
\hline Gás & 0,000000 & 0,000000 & 0,000009 \\
\hline Onda & 0,000000 & 0,000000 & 0,000000 \\
\hline Interação & 0,000000 & 0,000440 & 0,000000 \\
\hline
\end{tabular}

\section{Conclusões}

A partir dos resultados obtidos é possível concluir que:

- A adição de $\mathrm{CO}_{2}$ no gás de proteção reduz a convexidade do cordão, contudo prejudica o aspecto superficial;

- O perfil do reforço e largura, em geral podem ser melhorados (reduzindo a convexidade), com o aumento do potencial de oxigênio da mistura gasosa e/ou com a utilização do formato de onda do tipo $\mathrm{C}$ que possui base positiva antes do pulso de destacamento, alcançando nestas condições maior molhabilidade; 
- Os maiores valores de rendimento de deposição do processo MIG/MAG-PV nos gases estudados foram obtidos nas condições onde se utilizou o formato de onda $\mathrm{C}$, confirmando sua maior estabilidade e regularidade na transferência metálica;

- Independente das forças repulsivas geradas quando da utilização de $\mathrm{CO}_{2}$, elevados valores de rendimento de deposição foram obtidos para a mistura $\mathrm{Ar}+8 \% \mathrm{CO}_{2}$. Além disto, mesmo em $15 \% \mathrm{CO}_{2}$ resultados satisfatórios de rendimento de deposição foram encontrados.

\section{Agradecimentos}

Os autores agradecem ao Laprosolda/UFU pela disponibilidade de pessoal e estrutura física, à Fapemig pelo apoio financeiro para o comparecimento ao evento, à CAPES pelo financiamento da bolsa de estudos e ao CNPq (Processos 473953/20099 e 307554/2008-2).

\section{Referências Bibliográficas}

[1] HARADA, S; et al. The State-of-the Art of AC GMAW Process in Japan. IIW Doc. XII-1589-99. July 1999.

[2] SCOTTI, A; PONOMAREV, V; Soldagem MIG/MAG: Melhor Entendimento, Melhor Desempenho; São Paulo: Artliber Editora, 2008.

[3] TONG, H.; et al. Quality and Productivity Improvement in Aluminium Alloy Thin Sheet Welding Using Alternating Current Pulsed Metal Inert Gas Welding System. Science and Technology of Welding and Joining, v. 6, p. 203-208. 2001.

[4] UEYAMA, T; et al. AC Pulsed GMAW Improves Sheet Metal Joining. Welding Journal. February, 2005.

[5] NORRISH, J. Advanced Welding Process, 375pg, Cranfield Institute of Technology, p. 147, 1992.

[6] KARADENIZ, E; OZSARAC, U; YILDIZ, C. The Effect of Process Parameters on Penetration in Gas Metal Arc Welding Processes; Materials and Design 28, p. 649-656, 2007.

[7] NASCIMENTO, S. A. Fenomenologia da Soldagem MIG/ MAG-PV e sua Aplicabilidade para Juntas Sobreposta e em V (Passe de Raiz). 2011. 185f. Tese de doutorado, Universidade Federal de Uberlândia, Uberlândia.

[8] VILARINHO, L. O. et al . Methodology for Parameter Calculation of VP-GMAW. Welding Journal, v. Abril, p. 92s-98s, 2009.

[9] NASCIMENTO, A. S.; et al. Metodologia para Determinação de Parâmetros para Soldagem MIG com Polaridade Variável. Soldagem \& Inspeção, v. 14, p. 99-105, 2008.

[10] NASCIMENTO, A.S.; MENEZES JUNIOR, L.C.; VILARINHO, L.O. Características Cinemáticas da Transferência Metálica durante a Soldagem MIG/MAG-PV. Submetido ao Consolda 2011. Natal-RN, 11p., 2011.

[11] PIRES, I; QUINTINO, L; MIRANDA, R. M. Analysis of the Influence of Shielding Gas Mixtures on the Gas Metal Arc Welding Metal Transfer Modes and Fume Formation Rate. Materials and Design. 28, 1623-1631, 2007.

[12] AGA, Gas Handbook, 582 p, 1985.

[13] TALKINGTON, J. E. Variable Polarity Gas Metal Arc Welding: 1998. 126p. MSc Thesis - Ohio State University, USA.
[14] JELMORINI, G; TICHELAAR, G. W; Van den Heuvel, G. J. P. M. Droplet Temperature Measurements in Arc Welding. IIW Doc. 212-411-77, 22p, 1977.

[15] YAMAZAKI, K; et al; The measurement of metal droplet temperature in GMA welding by infrared two-colour pyrometry. Welding International, Volume 24, No 2, p. 81-87, 2010. 